\title{
A NEW SIX-DOF PARALLEL MECHANISM FOR CAPTIVE MODEL TEST
}

\author{
Yun Lu \\ Jinbo Wu \\ Weijia Li \\ Yaozhong Wu \\ School of Naval Architecture and Ocean Engineering, \\ Huazhong University of Science and Technology, China
}

\begin{abstract}
In order to obtain the hydrodynamic coefficients that can save cost and meet the accuracy requirements, a new hydrodynamic test platform based on a $6 \mathrm{DoF}$ (six degrees of freedom) parallel mechanism is proposed in this paper. The test platform can drive the ship to move in six degrees of freedom. By using this experimental platform, the corresponding hydrodynamic coefficients can be measured. Firstly, the structure of the new device is introduced. The working principle of the model is deduced based on the mathematical model. Then the hydrodynamic coefficients of a test ship model of a KELC tank ship with a scale of 1:150 are measured and 8 typical hydrodynamic coefficients are obtained. Finally, the measured data are compared with the value of a real ship. The deviation is less than $10 \%$ which meets the technical requirements of the practical project. The efficiency of measuring the hydrodynamic coefficients of physical models of ships and offshore structures is improved by the device. The method of measuring the hydrodynamic coefficients by using the proposed platform provides a certain reference for predicting the hydrodynamic performance of ships and offshore structures.
\end{abstract}

Keywords: hydrodynamic coefficient, six DoF parallel mechanism, six component balance, captive model test

\section{INTRODUCTION}

The hydrodynamic coefficients are the coefficients of the equations of motion of a ship or submersible. Therefore, the hydrodynamic coefficients must be determined first to simulate the manoeuvring motion and predict the manoeuvrability based on the ship manoeuvring motion equation [1-2]. At present, there are three main methods to obtain the hydrodynamic coefficients: the captive or free self-propelled model test, computer numerical simulation, and semi-theoretical and semi-empirical estimation [3-5].

Hydrodynamic model tests can be divided into captive model tests and free self-propelled ship model tests. There is a "scale effect" in the free self-propelled ship model test and it is still difficult to solve this problem at present. So the main method used is captive ship model testing, which mainly includes the oblique running test (ORT) [6], which is also known as the straight-line towing test, rotating arm test (RAT) [7], and plane motion mechanism (PMM) test [8-9].

The position derivative and coupling hydrodynamic coefficients of the submersible in the state of drift angle and rudder angle can be determined by the oblique running test. The force or moment derivatives related to angular velocity can be determined by the rotating arm test. At present, many scholars have obtained the corresponding hydrodynamic coefficients through these two tests. In order to study the influence of the free surface on the resistance and lift coefficient of a submersible, Mansoorzadeh et al. [10] used 
a 1:1 ship model to conduct a straight-line towing test in the towing pool and obtained the hydrodynamic coefficients of the submersible at different speeds and depths. Then they further studied the influence of the free surface on the resistance and lift coefficient. In order to research the hydrodynamic performance of small submersibles in the preliminary design, Gala et al. [11] obtained the hydrodynamic coefficients of small submersibles at different drift angles and attack angles using the ORT test and numerical simulation methods. They compared the results obtained by the two methods to verify the feasibility of the numerical method. For the same purpose, Li Gang et al. [12] took a certain submersible as a model to carry out the rotating arm test to obtain the rotating derivative, and used the data obtained to verify the accuracy of the numerical rotating arm pool model.

Avila et al. [13] conducted pure sway and pure yaw motion tests with a full-scale open frame submersible through a PMM to obtain the linear acceleration derivative and linear velocity derivative, thus providing sufficient hydrodynamic derivatives for manoeuvrability prediction. However, the simulation of pure sway and pure head roll can only obtain the inertial hydrodynamic coefficients of the horizontal plane, but not the vertical plane. Therefore, Xu et al. [14] conducted pure heave and pure pitch motion tests through a vertical plane motion mechanism and circulation tank, and obtained the inertial hydrodynamic coefficients of the vertical plane of the submersible. At present, more scholars are using the plane motion mechanism to test and study the hydrodynamic force of the submersible under the condition of the $6 \mathrm{DoF}$ coupling motion, and the research results are quite abundant. For example, Zhao et al. [15] took a small submersible with a pod as the research object to conduct hydrodynamic model tests, such as direct navigation, slant navigation, pure heave, pure pitch, pure heave and pure yaw, etc., to obtain the hydrodynamic coefficients needed for submersible manoeuvrability prediction and to compare and verify the calculated results with the numerical results. Pang et al. [16] used a VPMM (vertical PMM) in the circulation tank to conduct constraint model tests on submarine models without and with oars, and analysed the influence of the propeller on the hydrodynamic manoeuvrability of the submarine.

With the development of computers, the computational fluid dynamics (CFD) method is increasingly applied to the estimation of hydrodynamic derivatives. In 1990, Kijima and Nakiri [17] proposed a hydrodynamic derivative calculation method for manoeuvring motion, considering the stern shape based on the test data of 15 boats. In 1999, Maekawa et al. [18] proposed a CFD method to calculate the additional mass factor of the superstructure part of the ship. In 2000, Kijima et al. [19] used the constrained model test to measure a damaged ship in different trim states and obtained its hydrodynamic data. In 2000, Petersen and Lauridsen [20] proposed a new regression method with higher accuracy and adaptability based on the hydrodynamic derivatives of manoeuvring motion obtained from a PMM test. In 2006, through CFD calculation and verification analysis of a series of ship models, Yang [21] proved that the hydrodynamic CFD prediction method for manoeuvrability of large ships had good accuracy and universality. In 2018, Gao et al. [22] proposed a space capture motion simulation method for determining the hydrodynamic model coefficients.

The Stewart platform originated from a six degrees of freedom flight simulation mechanism designed by Stewart [23], which simulates the flight state of an aircraft by generating $6 \mathrm{DoF}$ motion in space. The mechanism designed by Stewart is supported by a triangular platform. The spherical hinge is supported on three driving legs with adjustable lengths and angles, and connected to the ground through two axle joints. Yurt designed a Stewart platform with six degrees of freedom and a pneumatic actuator for a flight simulator by using the method of complex dynamic system modelling [24]. Landry designed and implemented a low-cost flight simulator using commercial components for the Royal Military Academy of Canada [25]. Phoemsapthawee studied a new autonomous underwater vehicle by combining a $6 \mathrm{DoF}$ motion simulator with an unsteady potential flow coupling model [26]. Kim developed a six axis force/moment sensor based on the Stewart platform [27].

There are different ship models and many appendages. At present, there is no systematic design data available for approximate calculation. Therefore, the empirical equations of surface ships, submarines, torpedoes, etc. are often used. The estimation of the hydrodynamic force has brought great errors. Generally speaking, the semi-empirical and semi- theoretical estimation method can save time and capital cost, but it cannot meet the precision required in engineering $[28,29]$. The hydrodynamic coefficients obtained by the computer simulation method have high accuracy and can meet the requirements of manoeuvrability design, motion prediction and simulation in the preliminary design stage. However, the hydrodynamic coefficients that can be accurately calculated are fewer, and the calculation of the ship coupling hydrodynamic coefficients is still not accurate enough. Moreover, with the computer simulation method it is difficult to select the optimal model, decouple and identify it, and it cannot be applied to all ships. In addition, the method has some errors in calculating the coefficient of viscosity.

The captive model test is the most mature method to determine the hydrodynamic coefficients and it is still the main method used to solve practical problems in engineering. The test results are often compared with the numerical simulation results. However, the model test usually needs a lot of manpower and material resources and the test period is long. It is the focus of scholars to find a method to obtain the hydrodynamic coefficients that can not only save costs but also meet the accuracy requirements. The $6 \mathrm{DoF}$ parallel motion mechanism studied in this paper has become a promising method to solve the above problems. The $6 \mathrm{DoF}$ mechanism can generate $6 \mathrm{DoF}$ independent or coordinated motion in a single device, which is of great significance for the effective measurement of the hydrodynamic coefficients of complex motion physical models. 


\section{TEST MECHANISM AND THEORETICAL ANALYSIS}

\section{STRUCTURE OF SIX DOF PARALLEL MECHANISM MODEL}

The hydrodynamic test platform of the 6DoF parallel mechanism is composed of the $6 \mathrm{DoF}$ parallel mechanism, a six-dimensional force/torque sensor, a trailer, an ultrasonic rangefinder and a corresponding control system. The performance parameters of the test equipment are shown in Table 1 . Because the electric driven $6 \mathrm{DoF}$ platform is quiet, clean and precise, this research adopts an electrically driven way to make the $6 \mathrm{DoF}$ platform generate motion. The basic motion of the platform is the linear reciprocating motion of six moving rods driven by the electric cylinder driven by the servo motor. For better installation and control, the upper and lower planes of the $6 \mathrm{DoF}$ platform are a motion plane and a fixed plane respectively. The fixed plane is connected with the lower half of the trailer frame, while the motion plane is connected with the test ship model, and the connected part is equipped with the six-dimensional force/moment sensor. Fig. 1 shows the structure of the hydrodynamic test platform.

Tab. 1. Types of experiment platform

\begin{tabular}{|c|c|c|}
\hline Equipment & Type & $\begin{array}{c}\text { Technical } \\
\text { parameter }\end{array}$ \\
\hline $\begin{array}{c}\text { 6DoF parallel } \\
\text { mechanism }\end{array}$ & Motus TII & See Table 4 \\
\hline $\begin{array}{c}\text { Ultrasonic } \\
\text { rangefinder }\end{array}$ & UC2000-30GM-IUR2-V15 & $\begin{array}{c}\text { Sensing distance: } \\
80-2000 \mathrm{~mm}\end{array}$ \\
\hline $\begin{array}{c}\text { Six-dimensional } \\
\text { force/torque sensor }\end{array}$ & K6D68 2kN/50N•m & See Table 5 \\
\hline Control system & Adv. Tech. S30861 & - \\
\hline
\end{tabular}

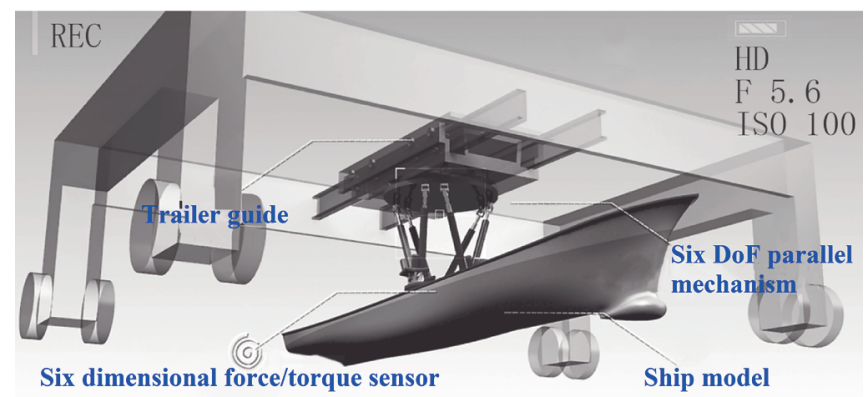

Fig. 1. Structure of the hydrodynamic test platform

The function of the $6 \mathrm{DoF}$ platform is embodied in the movement of six degrees of freedom. The motion is composed of the linear motion of six moving bars, including three displacements and three motions. The motion of these six degrees of freedom is essentially the same as that of the ship, i.e. roll, pitch, yaw, sway, surge and heave. In general, in order for the test device to drive the ship model to move according to the predetermined values of roll angle, pitch angle, yaw angle, sway displacement, surge displacement and heave displacement, it is necessary to give motion instructions to all the moving rods of the $6 \mathrm{DoF}$ platform, i.e. to input six linear displacements, and output them to the ship's roll, pitch, yaw, sway, surge and heave after conversion. The working principle is elaborated in the second and third parts of this section.

\section{KINEMATICS ANALYSIS OF SIX-DOF PARALLEL MECHANISM}

The terminal manoeuvring platform forces the experimental ship model to perform specific motions. Therefore, we need to calculate the motion relation between the input variables of the $6 \mathrm{DoF}$ parallel mechanism and the experimental ship model. The aim of kinematics analysis is mainly to establish the relationship between the pose of the manoeuvring platform and the input variables. Kinematics analysis is the basis of force analysis, workspace calculation, dynamics analysis, and robot motion planning and control.

For kinematics analysis, we define two right hand Cartesian coordinates which are shown in Fig. 2. $\{\mathbf{B}\}$ is the coordinate point vector of the upper control platform and its corresponding coordinate system $\mathrm{O}-\mathrm{X}_{\mathrm{B}} \mathrm{Y}_{\mathrm{B}} \mathrm{Z}_{\mathrm{B}}$ is a fixed coordinate system. $\{\mathbf{b}\}$ is the coordinate point vector of the bearing hinge point in the lower control platform and its corresponding coordinate system $\mathrm{O}-\mathrm{X}_{\mathrm{b}} \mathrm{Y}_{\mathrm{b}} \mathrm{Z}_{\mathrm{b}}$ is a moving coordinate system.

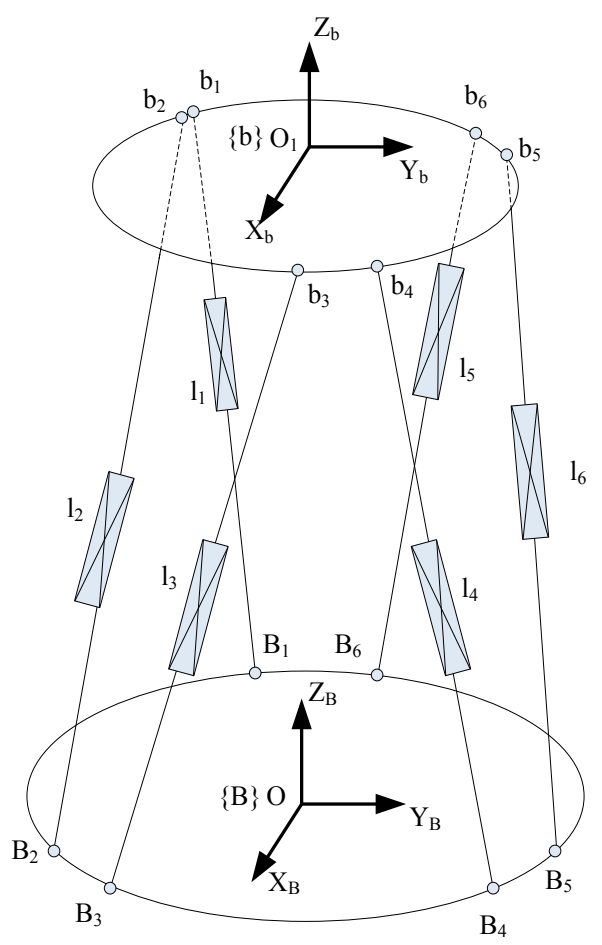

Fig. 2. Coordinate transformation of six-DoF parallel mechanism in two kinds of right-handed Cartesian coordinates

The rotation transformation of the platform occurs relative to axes $\mathrm{X}, \mathrm{Y}$ and $\mathrm{Z}$ with the angles of $\alpha, \beta$ and $\gamma$, respectively, which is shown in the schematic diagram of the platform position (Fig. 3) and platform movement posture (Fig. 4). Using the rotation representation of the Euler angle, 
Eqs. (1)-(3) can be obtained according to the homogeneous coordinate transformation method.

$$
\begin{aligned}
& \operatorname{Rot}(x, \alpha)=R_{1}=\left[\begin{array}{cccc}
1 & 0 & 0 & 0 \\
0 & \cos \alpha & -\sin \alpha & 0 \\
0 & \sin \alpha & \cos \alpha & 0 \\
0 & 0 & 0 & 1
\end{array}\right] \\
& \operatorname{Rot}(y, \beta)=R_{2}=\left[\begin{array}{cccc}
\cos \beta & 0 & \sin \beta & 0 \\
0 & 1 & 0 & 0 \\
-\sin \beta & 0 & \cos \beta & 0 \\
0 & 0 & 0 & 1
\end{array}\right] \\
& \operatorname{Rot}(z, \gamma)=R_{3}=\left[\begin{array}{cccc}
\cos \gamma & -\sin \gamma & 0 & 0 \\
\sin \gamma & \cos \gamma & 0 & 0 \\
0 & 0 & 1 & 0 \\
0 & 0 & 0 & 1
\end{array}\right]
\end{aligned}
$$

The translation transformation can be described as:

$$
\operatorname{Trans}(a, b, c)=\left[\begin{array}{cccc}
1 & 0 & 0 & a \\
0 & 1 & 0 & b \\
0 & 0 & 1 & c \\
0 & 0 & 0 & 1
\end{array}\right]
$$

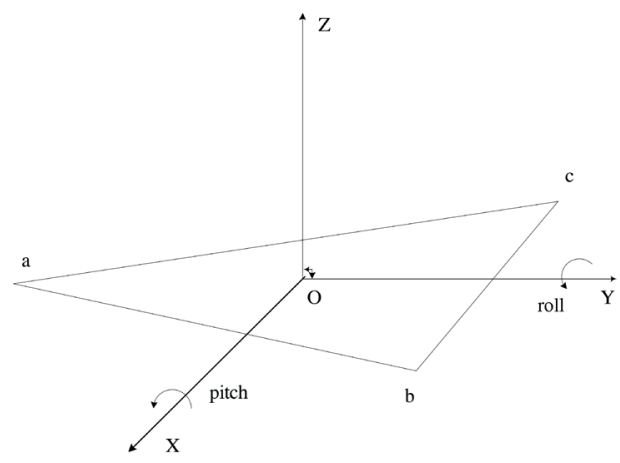

Fig. 3. Schematic diagram of platform position

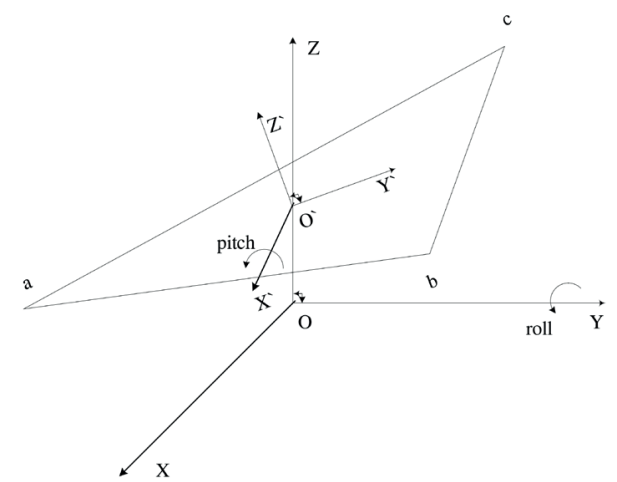

Fig. 4. Schematic diagram of platform movement posture
The coordinate transformation matrix $\mathrm{T}_{\mathrm{Bb}}$ of coordinate point $\{\mathbf{b}\}$ in the moving coordinate system with respect to point $\{\mathbf{B}\}$ in the fixed coordinate system can be described as:

$$
T_{B b}=\left[\begin{array}{cc}
R_{B b} & P_{B b} \\
0 & 1
\end{array}\right]
$$

In Eq. (5):

$\mathrm{R}_{\mathrm{Bb}}$ - rotation matrix of the spindle direction of the moving system $\{\mathbf{b}\}$ relative to the fixed system $\{\mathbf{B}\}$;

$\mathbf{P}_{\mathrm{Bb}}$ - position vector of the coordinate origin of the moving system $\{\mathbf{b}\}$ in the fixed system $\{\mathbf{B}\}$,

where:

$$
\begin{gathered}
R_{B b}(\alpha, \beta, \gamma)=\operatorname{Rot}(x, \alpha) \operatorname{Rot}(y, \beta) \operatorname{Rot}(z, \gamma) \\
\mathbf{P}_{\mathbf{B b}}=[x, y, z]^{T}
\end{gathered}
$$

$\mathbf{X}=[x, y, z, \alpha, \beta, \gamma]^{T}$ is the spatial pose of a point in $\{\mathbf{b}\}$ relative to $\{\mathbf{B}\}$ which is described by six-dimensional column vectors. Here, $\mathbf{b}_{\mathbf{i} \mathbf{M}}$ is the pose vector while $\alpha, \beta$ and $\gamma$ are Euler angles of $\{\mathbf{b}\}$. Any point $b_{\mathrm{iM}}$ on $\{\mathbf{b}\}$ can be expressed in the fixed coordinate system $\{\mathbf{B}\}$ as:

$$
\mathbf{b}_{\mathbf{i}}=T_{B b} \mathbf{b}_{\mathrm{iM}}
$$

For the given parallel mechanism and the corresponding structure size, as well as the selected $\{\mathbf{B}\}$ and $\{\mathbf{b}\}$, the coordinate values $\left(\mathbf{b}_{\mathbf{i}}\right.$ and $\left.\mathbf{B}_{\mathbf{i}}, \mathbf{i}=1,2, \ldots, 6\right)$ of each hinge point in the upper and lower platform in their respective coordinate systems can be calculated according to the geometric relations. For the given pose $\mathrm{q}=\left(\mathrm{P}_{\mathrm{Bb}}, \mathrm{R}_{\mathrm{Bb}}\right)$ of the upper platform mechanism, the coordinate value of each hinge point of the upper platform in $\{\mathbf{B}\}$ can be calculated from Eq. (6) as follows:

$$
\mathbf{b}_{\mathbf{i}}=R_{B b} \mathbf{b}_{\mathrm{iM}}+\mathbf{P}_{\mathrm{Bb}}, \quad i=1, \quad 2, \quad \ldots, 6
$$

Thus, the length vector $\mathbf{1}_{\mathrm{i}}, \mathrm{i}=1,2, \ldots, 6$ of the six driving cylinder rods in the global coordinate system can be expressed as:

$$
\mathbf{1}_{\mathbf{i}}=\mathbf{b}_{\mathbf{i}}-\mathbf{B}_{\mathbf{i}}=R_{B b} \mathbf{b}_{\mathrm{iM}}+\mathbf{P}_{\mathrm{Bb}}-\mathbf{B}_{\mathbf{i}}
$$

The length of a single leg can be expressed as the Eqs. (9):

$$
\begin{aligned}
l_{i} & =\left|\mathbf{1}_{\mathbf{i}}\right|=f_{i}(x)=\sqrt{\mathbf{1}_{\mathbf{i}}^{\mathbf{T}} \mathbf{1}_{\mathbf{i}}} \\
& =\sqrt{\left(R_{B b} \mathbf{b}_{\mathrm{iM}}+\mathbf{P}_{\mathrm{Bb}}-\mathbf{B}_{\mathbf{i}}\right)^{T}\left(R_{B b} \mathbf{b}_{\mathbf{i M}}+\mathbf{P}_{\mathbf{B b}}-\mathbf{B}_{\mathbf{i}}\right)}
\end{aligned}
$$


If the initial length of the leg is $l_{i 0}$, the elongation of the leg can be expressed as:

$$
\Delta l_{i}=l_{i}-l_{i 0}
$$

The Jacobian matrix represents the mapping from joint velocity to operational velocity, and $\mathrm{J}(\mathrm{x})$ can be noted as the Jacobian matrix in position $\mathrm{x}$. By fully differentiating the vector Eq. (9), the Jacobian matrix $\mathrm{H}(\mathrm{x})$ of the parallel mechanism can be obtained.

$d l=\left[\begin{array}{c}d l_{1} \\ \ldots \\ d l_{6}\end{array}\right]=\left[\begin{array}{c}d f_{1}(x) \\ \ldots \\ d f_{6}(x)\end{array}\right]=\left[\begin{array}{ccc}\frac{\partial f_{1}}{\partial x_{1}} & \ldots & \frac{\partial f_{1}}{\partial x_{6}} \\ \ldots & \ldots & \ldots \\ \frac{\partial f_{6}}{\partial x_{1}} & \ldots & \frac{\partial f_{6}}{\partial x_{6}}\end{array}\right]\left[\begin{array}{c}d x_{1} \\ \ldots \\ d x_{6}\end{array}\right]=H(x) d x$

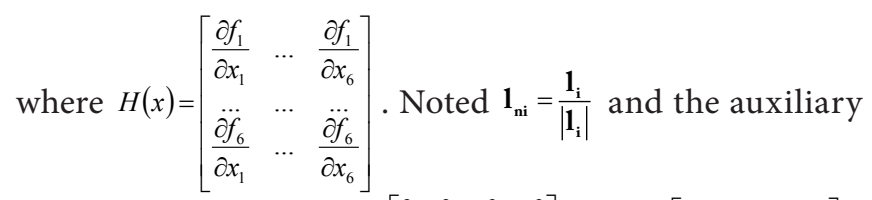
matrix $K=\left[\begin{array}{c}I_{3} \\ 0\end{array}\right]_{4 \times 3}, \quad K_{1}=\left[\begin{array}{cccc}0 & 0 & 0 & 0 \\ 0 & 0 & -1 & 0 \\ 0 & 1 & 0 & 0 \\ 0 & 0 & 0 & 1\end{array}\right], \quad K_{2}=\left[\begin{array}{cccc}0 & 0 & 1 & 0 \\ 0 & 0 & 0 & 0 \\ -1 & 0 & 0 & 0 \\ 0 & 0 & 0 & 1\end{array}\right]$, $K_{3}=\left[\begin{array}{cccc}0 & -1 & 0 & 0 \\ 1 & 0 & 0 & 0 \\ 0 & 0 & 0 & 0 \\ 0 & 0 & 0 & 1\end{array}\right]$

By taking the derivative with respect to time for Eq. (7), we can get Eq. (12):

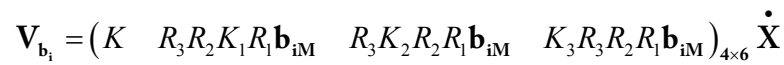

The velocity matrix of each driving leg can be written as Eq. (13) as follows:

$$
\dot{1}_{i}=\frac{1_{i}}{\left|1_{i}\right|} V_{b_{i}}=1_{n i} V_{b_{i}}
$$

The velocity vector of the six driving legs can be written as $\dot{i}_{=}=\left[\begin{array}{llllll}\dot{i}_{1} & \dot{i}_{2} & \dot{i}_{3} & \dot{i}_{4} & \dot{i}_{5} & \dot{i}_{6}\end{array}\right]$, then we can get the Eqs. (14):

$$
\mathrm{i}=\left[\begin{array}{lllllll}
1_{n 1} V_{b_{1}} & 1_{n 2} V_{b_{2}} & 1_{n 3} V_{b_{3}} & 1_{n 4} V_{b_{4}} & 1_{n 5} V_{b_{5}} & 1_{n 6} V_{b_{6}}
\end{array}\right]
$$

Eq. (14) reflects the generalised velocity vectors between each drive leg and the upper manoeuvring platform. By taking the derivative with respect to time for Eq. (14), we can get Eq. (15), which reflects the generalised acceleration vectors between each drive leg and the upper manoeuvring platform.

$$
\ddot{\mathrm{i}}=\left[\begin{array}{llll}
\dot{\mathrm{i}}_{\mathrm{n} 1} \mathrm{~V}_{\mathrm{b}_{1}}+\mathbf{1}_{\mathrm{n} 1} \dot{\mathrm{V}}_{\mathrm{b}_{1}} & \dot{\mathrm{i}}_{\mathrm{n} 2} \mathrm{~V}_{\mathrm{b}_{2}}+\mathbf{1}_{\mathrm{n} 2} \dot{\mathrm{V}}_{\mathrm{b}_{2}} \quad \ldots \quad \dot{\mathrm{i}}_{\mathrm{n} 6} \mathrm{~V}_{\mathrm{b}_{6}}+\mathbf{1}_{\mathrm{n} 6} \dot{\mathrm{V}}_{\mathrm{b}_{6}}
\end{array}\right]
$$

The upper manoeuvring platform is connected with the experimental ship model through a rigid two-force bar, so the motion of the upper manoeuvring platform can be regarded as the motion of the experimental ship model. From Eqs. (9)(10) and (14)-(15), the displacement, velocity and acceleration relations between the six driving legs and the ship model are established. Once the theoretical motion of the ship model is given, the corresponding elongation of the driving legs can be calculated to drive the ship model to achieve the specified motion.

\section{THEORETICAL ANALYSIS OF MANOEUVRING HYDRODYNAMIC COEFFICIENT}

It is necessary to make some assumptions before establishing the manipulation motion equation, which can make it more convenient and rigorous for us to study the problem. Previous studies have shown that these assumptions have little influence on the hydrodynamic characteristics and manipulation performance. These assumptions include the following:

1) The ship is a rigid body with constant mass and its geometric shape does not change. The actual situation is that due to the movement of the internal structure and movement of persons, the mass and centroid of the ship are changing, such as the change of mass and centroid caused by ballast abandonment or ballast tank drainage. The influence of these changes on the hydrodynamic force is no longer within the scope of this paper.

2) The reference frame (definite frame) is the Earth, and its rotational motion effect is ignored.

3) The only forces acting on the manned deep submersible are gravity, buoyancy, hydrodynamic force and control force, and the other forces under special circumstances are not included in the scope of consideration, such as water entering the damaged cabin, being attacked, and the internal reaction of the working object, etc.

4) It is assumed that the dimensionless hydrodynamic coefficients are all constant in the study of hydrodynamic problems by means of an analytical method or Taylor expansion. This assumption is based on the "small disturbance", which has achieved great success for submarines. The dimensionless hydrodynamic changes are not considered in this paper.

5) In the analysis of hydrodynamic coefficients, the flow field around the manned submersible is assumed to be infinite and unaffected by the seabed and free surface.

A rectangular coordinate system is usually used to study ship motion. The basic coordinate system is a fixed coordinate system and a moving coordinate system. According to convention, the right hand system is adopted. The system of parameters and symbols used in the study of ship motion is generally recommended by the International Water Pool Conference and by the Shipbuilding and Marine Engineering Society. This system is also used in this study. The fixed 
coordinate system $\mathrm{E}-\xi \eta \zeta$ and moving coordinate system $\mathrm{O}-\mathrm{XYZ}$ are shown in Fig. 5.

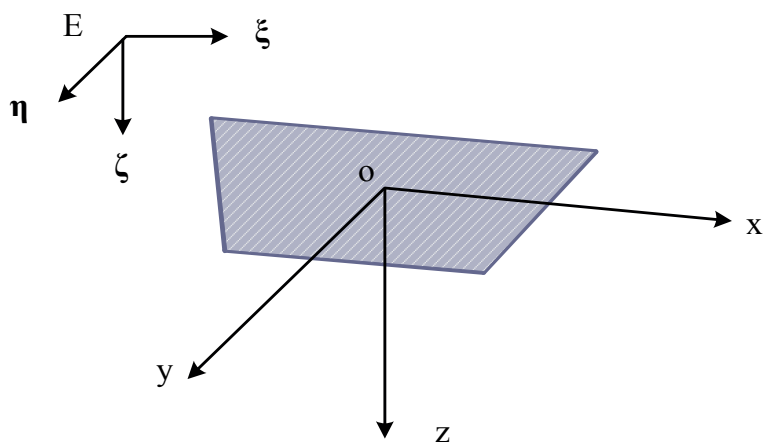

Fig. 5. The coordinate system

The description of the main symbols in the fixed coordinate system is given in Table 2 , and in the moving coordinate system, it is shown in Table 3. Assuming that the environmental condition is infinite water depth, according to Newton's motion theorem and the definition of the MMG model, the ship's 6 DoF operating motion equation can be expressed as follows:

$$
\left\{\begin{array}{l}
m(\dot{u}-v r+w q)=X \\
m(\dot{v}-p w+u r)=Y \\
m(\dot{w}-u q+v p)=Z \\
I_{x x} \dot{p}=K \\
I_{y y} \dot{q}+\left(I_{x x}-I_{z z}\right) p r=M \\
I_{z z} \dot{r}+\left(I_{y y}-I_{x x}\right) p q=N
\end{array}\right.
$$

In Eq. (16):

$\mathrm{m}$ - mass of the ship;

$I_{x x}, I_{y y}, I_{z z}$ - moment of inertia about the $\mathrm{X}, \mathrm{Y}$ and $\mathrm{Z}$ axes.

Tab. 2. Main symbols in the fixed coordinate system

\begin{tabular}{|c|c|c|c|}
\hline Points or vectors & $\xi$ axis & $\eta$ axis & $\zeta$ axis \\
\hline Centre of gravity,-G & $\xi_{\mathrm{G}}$ & $\eta_{G}$ & $\zeta_{\mathrm{G}}$ \\
\hline Origin, $\mathrm{o}$ & $\xi_{\mathrm{o}}$ & $\eta_{0}$ & $\zeta_{0}$ \\
\hline Velocity, U & $\mathrm{U}_{\xi}$ & $\mathrm{U}_{\eta}$ & $\mathrm{U}_{\zeta}$ \\
\hline Angular velocity, $\Omega$ & $\Omega_{\xi}$ & $\Omega_{\eta}$ & $\Omega_{\zeta}$ \\
\hline Force, F & $\mathrm{F}_{\xi}$ & $F_{\eta}$ & $\mathrm{F}_{\zeta}$ \\
\hline Torque, $\mathrm{T}$ & $\mathrm{T}_{\xi}$ & $\mathrm{T}_{\eta}$ & $\mathrm{T}_{\zeta}$ \\
\hline
\end{tabular}

Tab. 3. Main symbols in the moving coordinate system

\begin{tabular}{|l|c|c|c|}
\hline \multicolumn{1}{|c|}{ Vectors } & X axis & Y axis & Z axis \\
\hline Velocity, U & $\mathrm{u}$ & $\mathrm{v}$ & $\omega$ \\
\hline Angular velocity, $\Omega$ & $\mathrm{p}$ & $\mathrm{q}$ & $\mathrm{r}$ \\
\hline Force, $\mathrm{F}$ & $\mathrm{X}$ & $\mathrm{Y}$ & $\mathrm{Z}$ \\
\hline Torque, T & $\mathrm{K}$ & $\mathrm{M}$ & $\mathrm{N}$ \\
\hline
\end{tabular}

The hydrodynamic force of a ship can be expressed as a function of four major factors, namely, the flow field characteristics, the hull characteristics, the ship's motion state and the ship's manoeuvring factors. Before Taylor expansion of the hydrodynamics, the following points should be made clear: the flow field characteristics are variable elements, the ship motion state and control elements must change at any time and, on the contrary, the ship body keeps its inherent state unchanged. The hypothesis is shown as follows:

1) The flow field characteristics remain relatively unchanged for a period of time.

2) Only three degrees of freedom motion including direct flight, transversal drift and turning head are considered in the horizontal plane, and the motion parameters include (angular) velocity and (angular) acceleration.

3) The initial motion of the ship is assumed to be straight sailing at a constant speed.

4) The higher derivative is considered to be infinitesimal and the influence of parameter $a$ is minimal.

5) The hull moves in a small linear range. In practical application, the second-order increment of motion parameters can be ignored and the amplitude of motion parameters can be changed slightly according to specific requirements.

6) The linear proportional relationship between flow force and (angular) acceleration is established. The expansion does not include the higher-order term and the resultant term of (angular) acceleration.

7) The coupling in all directions is ignored.

8) The ship is symmetrical left and right.

In conclusion, the hydrodynamic force $\mathrm{G}$ can be expressed as follows:

$$
G=G\left(u, v, r, \dot{u}, \dot{v}, \dot{r}, \delta_{r}\right)
$$

where $\delta_{r}$ is the rudder angle. According to the above hypothesis, after Taylor expansion, the following results can be obtained:

$G=G_{0}+G_{\dot{u}} \dot{u}+G_{\dot{v}} \dot{v}+G_{\dot{r}} \dot{r}+G_{u}\left(u-u_{0}\right)+G_{v} v+G_{r} r+G_{\delta_{r}} \delta_{r}$

where $G_{0}, G_{\dot{u}}, G_{\dot{v}}, G_{\dot{r}}, G_{u}, G_{v}, G_{r}, G_{\delta_{r}}$ are the hydrodynamic coefficients. Taking $G_{\dot{u}}$ as an example, its specific form is as follows:

$$
G_{\dot{u}}=\left.\frac{\partial G}{\partial \dot{u}}\right|_{\substack{u_{0}=V \\ v_{0}=r_{0}=\dot{u}_{0}=\dot{v}_{0}=\dot{r}_{0}=\delta_{r 0}=0}}
$$

These hydrodynamic coefficients have practical physical meaning. They respectively represent the force in the direction in which the velocity or acceleration is generated. In Eq. (18), the zero-order term represents the inherent hydrodynamic force of a ship in a straight voyage of uniform speed, while the first-order term is all the hydrodynamic force caused by speed or acceleration and exists in the form of increments. Therefore, the first-order term can be regarded as the modification of the zero-order hydrodynamic force. 
In this paper, the horizontal plane motion model is established. By taking $\mathrm{X}, \mathrm{Y}$ and $\mathrm{N}$ into different motion directions, the hydrodynamic expression of the model is derived:

$\left\{\begin{array}{l}X=X_{0}+X_{\dot{u}} \dot{u}+X_{\dot{v}} \dot{v}+X_{\dot{r}} \dot{r}+X_{u}\left(u-u_{0}\right)+X_{v} v+X_{r} r+X_{\delta_{r}} \delta_{r} \\ Y=Y_{0}+Y_{\dot{u}} \dot{u}+Y_{\dot{v}} \dot{v}+Y_{\dot{r}} \dot{r}+Y_{u}\left(u-u_{0}\right)+Y_{v} v+Y_{r} r+Y_{\delta_{r}} \delta_{r} \\ N=N_{0}+N_{\dot{u}} \dot{u}+N_{\dot{v}} \dot{v}+N_{\dot{r}} \dot{r}+N_{u}\left(u-u_{0}\right)+N_{v} v+N_{r} r+N_{\delta_{r}} \delta_{r}\end{array}\right.$

In the above equation, the velocity term is the fluid inertial force, and the inertial force term is only related to the acceleration primary term. The viscous force of fluid is related to the properties of velocity and flow field. The lowerorder velocity term may contain both the inertial force term and the viscous force term. However, in the higher-order model, the third and higher degree terms of velocity only represent the viscous force.

\section{HYDRODYNAMIC TEST PLATFORM}

The main equipment of the $6 \mathrm{DoF}$ parallel mechanism test platform includes the $6 \mathrm{DoF}$ parallel mechanism, sixdimensional force/torque sensor and ultrasonic distance meter. The 6 DoF parallel mechanism is shown in Fig. 6, and its kinematic performance index is shown in Table 4. The six-dimensional force/torque sensor is FC-K6D68 from Germany, and its performance parameters are shown in Table 5. According to the theoretical calculation, the model of the six-component balance should be K6D68 2 kN/50 Nm.

Tab. 4. Kinematic performance index of $6 \mathrm{DoF}$ parallel mechanism

\begin{tabular}{|c|c|c|c|c|}
\hline Posture & Displacement & $\begin{array}{c}\text { (Angular) } \\
\text { Velocity }\end{array}$ & $\begin{array}{c}\text { (Angular) } \\
\text { Acceleration }\end{array}$ & $\begin{array}{c}\text { Oscillation } \\
\text { frequency } \\
(\mathbf{H z})\end{array}$ \\
\hline Roll & \pm 20 & $10-32 / \mathrm{s}$ & $5-51 / \mathrm{s}^{2}$ & $0.5-1.6$ \\
\hline Pitch & \pm 5 & $2.5-8 / \mathrm{s}$ & $1.3-13 / \mathrm{s}^{2}$ & $0.5-1.6$ \\
\hline Yaw & \pm 15 & $7.5-24 / \mathrm{s}$ & $3.8-38 / \mathrm{s}^{2}$ & $0.5-1.6$ \\
\hline Surge & $\pm 150 \mathrm{~mm}$ & $75-240 \mathrm{~mm} / \mathrm{s}$ & $38-384 \mathrm{~mm} / \mathrm{s}^{2}$ & $0.5-1.6$ \\
\hline Sway & $\pm 150 \mathrm{~mm}$ & $75-240 \mathrm{~mm} / \mathrm{s}$ & $38-384 \mathrm{~mm} / \mathrm{s}^{2}$ & $0.5-1.6$ \\
\hline Heave & $\pm 100 \mathrm{~mm}$ & $50-160 \mathrm{~mm} / \mathrm{s}$ & $26-256 \mathrm{~mm} / \mathrm{s}^{2}$ & $0.5-1.6$ \\
\hline
\end{tabular}

Tab. 5. Performance parameters of the sensors

\begin{tabular}{|c|c|c|c|c|c|c|}
\hline Type & $\sum_{I^{*}}^{Z}$ & $\underbrace{Z}_{I^{2}}$ & $\sum_{I^{N}}^{\mathrm{Z}}$ & $\begin{array}{c}\Xi \\
\dot{z} \\
\sum^{*}\end{array}$ & 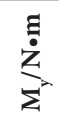 & $\frac{\Xi}{z^{\prime}}$ \\
\hline K6D68 $1 \mathrm{kN} / 20 \mathrm{~N} \bullet \mathrm{m}$ & 1 & 1 & 2 & 20 & 20 & 20 \\
\hline K6D68 2 kN/50 N•m & 2 & 2 & 4 & 50 & 50 & 50 \\
\hline K6D68 $5 \mathrm{kN} / 50 \mathrm{~N} \bullet \mathrm{m}$ & 5 & 5 & 10 & 50 & 50 & 50 \\
\hline K6D68 $10 \mathrm{kN} / 100 \mathrm{~N} \bullet \mathrm{m}$ & 10 & 10 & 20 & 100 & 100 & 100 \\
\hline $\mathrm{K} 6 \mathrm{D} 6810 \mathrm{kN} / 500 \mathrm{~N} \cdot \mathrm{m}$ & 10 & 10 & 20 & 500 & 500 & 500 \\
\hline
\end{tabular}

In order to ensure the stability of the box structure and full contact between the towing frame and the $6 \mathrm{DoF}$ platform during assembly, a stiffener structure is set on the right side of the lower half of the towing frame. Finally, limited by space, the 6 DoF platform can only be connected with the lower half of the trailer frame by hoisting from the side near the pool of the trailer frame platform. In order to facilitate the installation, a deep inverted trapezoid key-way is cut horizontally on the bottom steel plate of the lower half. Considering the positioning accuracy during the installation, the length of the key-way is less than the width of the steel plate. As the trailer frame is closely connected with the crossbeam on the trailer platform, the cross-section of the crossbeam is shown in Figure 7.

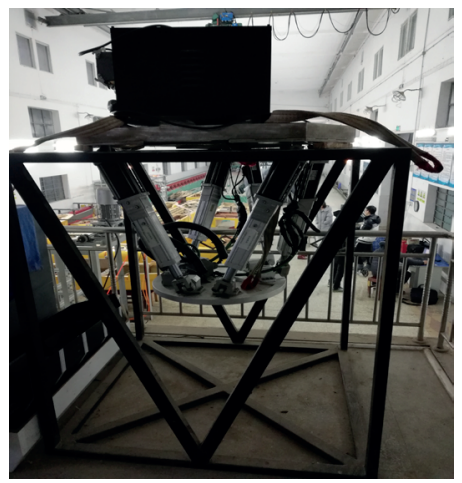

Fig. 6. The 6 DoF parallel mechanism.

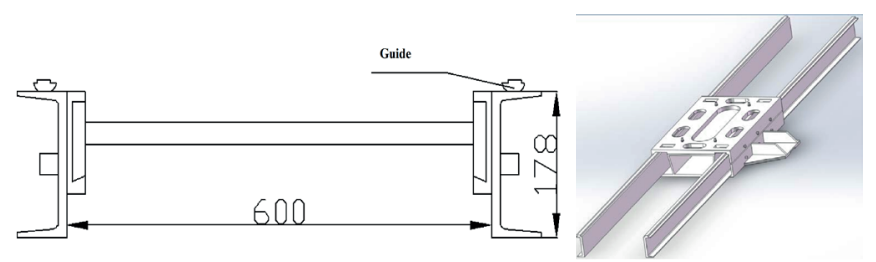

Fig. 7. The cross-beam and trailer rack.

The test ship model of the KELC tank ship designed by China Ship Scientific Research Centre (CSSRC) with a scale of 1:150 is used in the experiment. The basic parameters of the ship model on the liquid surface used in the test are shown in Table 6.

Tab. 6. Main parameters of the ship model

\begin{tabular}{|l|c|}
\hline \multicolumn{1}{|c|}{ Main parameter } & Value \\
\hline Length $(\mathrm{m})$ & 1.347 \\
\hline Moulded breadth $(\mathrm{m})$ & 0.30 \\
\hline Moulded depth $(\mathrm{m})$ & 0.29 \\
\hline Design draft $(\mathrm{m})$ & 0.04 \\
\hline Drainage quality $(\mathrm{kg})$ & 13.75 \\
\hline Maximum cross-sectional area $\left(\mathrm{S}_{\mathrm{M}} / \mathrm{m}_{2}\right)$ & 0.11 \\
\hline Focus & $(0.02,0,0)$ \\
\hline Moment of inertia $\mathrm{I}_{\mathrm{x}}, \mathrm{I}_{\mathrm{y}}, \mathrm{I}_{\mathrm{z}}\left(\mathrm{kg} \cdot \mathrm{m}^{2}\right)$ & $3.52,128.36,135.1$ \\
\hline
\end{tabular}

\section{EXPERIMENT RESULTS AND ANALYSIS}

In order to measure the hydrodynamic coefficients of the test ship model, captive model tests were carried out on the test ship model by using the $6 \mathrm{DoF}$ parallel mechanism test 
device. The steady-state test of the captive model includes an oblique running test and rudder angle test. The simple harmonic motion test of the captive model includes a sway test and yaw test. The hydrodynamic coefficients of the ship model were collected and analysed. The experiment was carried out in the Ship Model Towing Tank Laboratory (SMTTL) of SNAOE in Huazhong University of Science and Technology. The tank, which is a member of the International Towing Tank Conference (ITTC), is $175 \mathrm{~m}$ long, $6 \mathrm{~m}$ wide and 4 $m$ deep. The influence of the free surface is not taken into account in this paper.

\section{OBLIQUE RUNNING TEST}

The oblique running test is a drift angle test. In this test, the equation of ship motion is shown as follows:

$$
\left\{\begin{array}{l}
u=u_{0} \cos \beta \\
v=-u_{0} \sin \beta \\
r=0 \mathrm{rad} / \mathrm{s} \\
\delta=0 \mathrm{rad}
\end{array}\right.
$$

where $u_{0}$ denotes the dragging velocity, which is set according to the "Similarity Principle"; $\beta$ denotes the drift angle. It can be seen from the above equation that when the drift angle $\beta$ changes, the transverse and longitudinal velocities will change correspondingly. By changing the drift angle $\beta$, a series of longitudinal force, transverse force and yaw moment under different drift angles can be measured. The curves of longitudinal force, transverse force and yaw moment with transverse velocity can be obtained. Then the corresponding hydrodynamic coefficients are calculated after data fitting.

By adjusting the angle in the horizontal plane of the sixDoF motion mechanism, the drift angle $\beta$ can be tested, where $\beta \in\left[-10^{\circ}, 10^{\circ}\right], \Delta \beta=1^{\circ}$. The six component forces are measured by the six-dimensional force/torque sensor. Considering the non-linear effect of the horizontal force and torque, non-linear fitting is adopted to obtain the linear velocity coefficients $Y_{v}^{\prime}, \quad N_{v}^{\prime}$. The water density $\rho=1 \times 10^{3} \mathrm{~kg} /$ $\mathrm{m}^{3}$ and dragging velocity $\mathrm{u}_{0}=1.2 \mathrm{~m} / \mathrm{s}$. The results for the hydrodynamic coefficients are summarised in Table 7 . The diagram of transverse force and yaw moment changes with speed is shown in Fig. 8.

Tab. 7. Hydrodynamic coefficients of the ship model in oblique running test

\begin{tabular}{|c|c|c|c|}
\hline $\begin{array}{c}\text { Hydrodynamic } \\
\text { coefficient }\end{array}$ & Test value $\times \mathbf{1 0}^{-2}$ & $\begin{array}{c}\text { Value from real } \\
\text { ship } \times \mathbf{1 0}^{-2}[\mathbf{3 0}]\end{array}$ & $\begin{array}{c}\text { Percentage of } \\
\text { difference/\% }\end{array}$ \\
\hline$N_{v}^{\prime}$ & -1.592 & -1.609 & $1.06 \%$ \\
\hline$Y_{v}^{\prime}$ & -6.201 & -6.394 & $3.02 \%$ \\
\hline
\end{tabular}

\section{RUDDER ANGLE TEST}

The rudder angle test is carried out by changing the rudder angle of the steering gear when the drift angle is zero, where $\delta_{r} \in\left[-30^{\circ}, 30^{\circ}\right], \quad \Delta \delta_{r}=2.5^{\circ}$. The corresponding hydrodynamic force acting on the hull is measured through the test, and the rudder angle coefficients $Y_{\delta_{r}}^{\prime}, \quad N_{\delta_{r}}^{\prime}$ are obtained by linear fitting within the stall angle range and dimensionless. The results are summarised in Table 8 . The test results of the rudder are shown in Fig. 9.

Tab. 8. Hydrodynamic coefficients of the ship model in rudder angle test

\begin{tabular}{|c|c|c|c|}
\hline $\begin{array}{c}\text { Hydrodynamic } \\
\text { coefficient }\end{array}$ & Test value $\times \mathbf{1 0}^{-2}$ & $\begin{array}{c}\text { Value from real } \\
\text { ship } \times \mathbf{1 0}^{-2}[\mathbf{3 0}]\end{array}$ & $\begin{array}{c}\text { Percentage of } \\
\text { difference } / \%\end{array}$ \\
\hline$Y_{\delta_{r}}^{\prime}$ & -0.694 & -0.712 & $2.53 \%$ \\
\hline$N_{\delta_{r}}^{\prime}$ & 0.371 & 0.381 & $2.62 \%$ \\
\hline
\end{tabular}

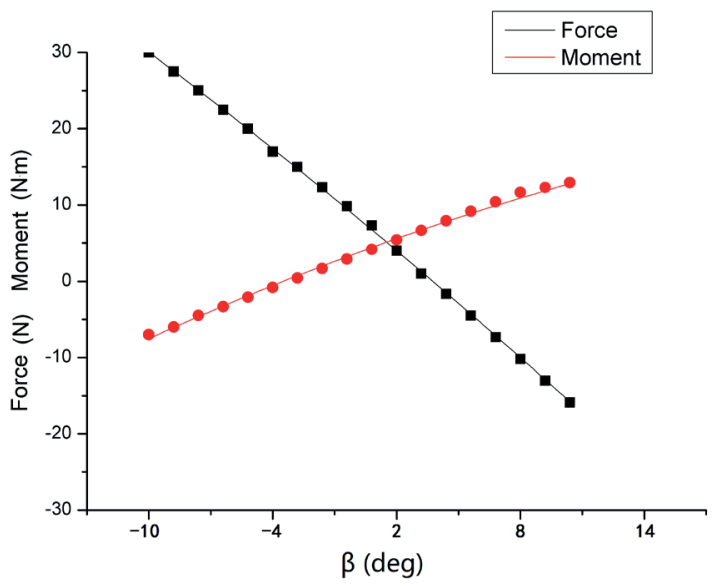

Fig. 8. The force and moment changes with speed

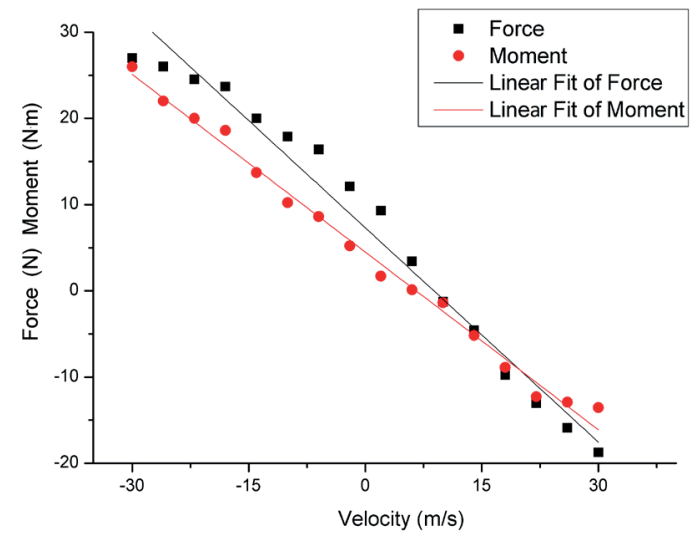

Fig. 9. The force and moment changes with rudder angle

\section{SWAY TEST}

The measured constraint force (moment) can be written in the form shown in Eq. (20) by means of motion decomposition, 
the inertial force (in-phase component) is represented by the subscript in, the damping force (orthogonal component) is represented by the subscript out, and the rest is constant.

$$
\left\{\begin{array}{l}
F_{x}(t)=X_{i} \sin (\omega t)+X_{\text {out }} \cos (\omega t)-Z_{0} \\
M_{y}(t)=M_{i} \sin (\omega t)+M_{\text {out }} \cos (\omega t)-M_{0}
\end{array}\right.
$$

When the ship model makes the transverse motion in the horizontal plane, the drift angle is always 0 . The motion parameters of the ship model are set as follows:

$$
\left\{\begin{array}{l}
y=a \sin (\omega t) \\
\psi=\dot{\psi}=0 \\
v=\dot{y}=a \omega \cos (\omega t) \\
\dot{v}=-a \omega^{2} \sin (\omega t)
\end{array}\right.
$$

In Eq. (21):

$y$ - transversal displacement of ship model;

a - amplitude of the motion;

$\omega$ - frequency of the motion;

$\psi, \quad \dot{\psi}$ - the tilt angle and angular velocity of the ship model around a vertical axis;

$v, \quad \dot{v}$ - transverse velocity and acceleration of the ship model.

Suppose that the force exerted on the object along the transverse direction is $\mathrm{Y}$ and the torque around the vertical axis is $\mathrm{N}$, then these two terms can be decomposed as follows according to the physical meaning of hydrodynamic force:

$$
\begin{aligned}
& Y=Y_{v} v+Y_{\dot{v}} \dot{v}+Y_{0} \\
& =-a \omega^{2} Y_{\dot{v}} \sin \omega t+a \omega Y_{v} \cos \omega t+Y_{0} \\
& N=N_{v} v+N_{\dot{v}} \dot{v}+N_{0} \\
& =-a \omega^{2} N_{\dot{v}} \sin \omega t+a \omega N_{v} \cos \omega t+N_{0}
\end{aligned}
$$

Obviously, the amplitude of the mass force (moment) $F_{\lambda}, M_{\lambda}$ and viscous force (moment) $F_{\mu}, M_{\mu}$ can be obtained by monitoring the change of force along the direction of motion with time. Then the hydrodynamic coefficient can be calculated as follows:

$$
\left\{\begin{array}{l}
Y_{\dot{v}}=-\frac{F_{\lambda}}{a \omega^{2}}, Y_{v}=\frac{F_{\mu}}{a \omega} \\
N_{\dot{v}}=-\frac{M_{\lambda}}{a \omega^{2}}, N_{v}=\frac{M_{\mu}}{a \omega}
\end{array}\right.
$$

Fig. 10 shows the sway motion of the test ship model. The change trend of the corresponding damping force and inertia force with the speed and acceleration is obtained through the test. The corresponding hydrodynamic coefficients can be obtained by linear fitting and combining the hydrodynamic model parameters in Table 6 into Eq. (24). The hydrodynamic coefficients obtained by the dimensionless treatment are summarised in Table 9. The test results are shown in Fig. 11.

Tab. 9. Hydrodynamic coefficients of the ship model in sway test

\begin{tabular}{|c|c|c|c|}
\hline $\begin{array}{c}\text { Hydrodynamic } \\
\text { coefficient }\end{array}$ & Test value $\times \mathbf{1 0}^{-2}$ & $\begin{array}{c}\text { Value from real } \\
\text { ship } \times \mathbf{1 0}^{-2}[\mathbf{3 0}]\end{array}$ & $\begin{array}{c}\text { Percentage of } \\
\text { difference/\% }\end{array}$ \\
\hline$Y_{\dot{v}}^{\prime}$ & -1.651 & -1.636 & $0.92 \%$ \\
\hline$Y_{v}^{\prime}$ & -6.108 & -6.394 & $4.68 \%$ \\
\hline$N_{\dot{v}}^{\prime}$ & -0.058 & -0.061 & $4.92 \%$ \\
\hline$N_{v}^{\prime}$ & -1.581 & -1.609 & $1.74 \%$ \\
\hline
\end{tabular}

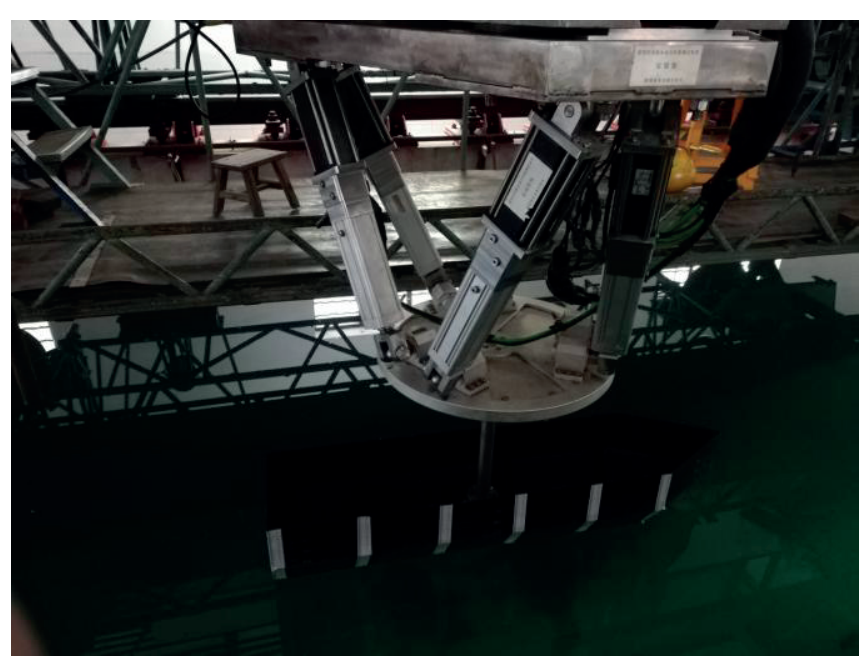

Fig. 10. The sway motion of the test ship model

\section{YAW TEST}

When the ship model makes a yaw motion in the horizontal plane, the incidence angle is always 0 . The motion parameters of the ship model are set as follows:

$$
\left\{\begin{array}{l}
\psi=\theta_{0} \cos (\omega t) \\
v=\dot{v}=0 \\
r=\dot{\psi}=-\theta_{0} \omega \sin (\omega t) \\
\dot{r}=-\theta_{0} \omega^{2} \cos (\omega t)
\end{array}\right.
$$

In Eq. (25):

$\theta_{0}$ - amplitude of yaw motion;

$\omega$ - frequency of yaw motion;

$r, \quad \dot{r}$ - the tilt angle and angular velocity of yaw motion. 
It needs to be noted that, in order to ensure that the centre line of the model is consistent with the direction of resultant velocity at all times, $\theta_{0}=-\frac{a \omega^{2}}{a}$, where $a$ is the amplitude of heave motion of the ship model; $\omega$ is the frequency of heave motion and $u$ denotes the inflow velocity.

Supposing that the force exerted on the object along the transverse direction is $\mathrm{Y}$ and the torque around the rotation axis is $\mathrm{N}$, we can get:

$$
\begin{aligned}
& Y=Y_{r} r+Y_{\dot{r}} \dot{r}+Y_{0} \\
& =-\theta_{0} \omega^{2} Y_{\dot{r}} \cos \omega t-\theta_{0} \omega Y_{r} \sin \omega t+Y_{0} \\
& N=N_{r} r+N_{\dot{r}} \dot{r}+N_{0} \\
& =-\theta_{0} \omega^{2} N_{\dot{r}} \cos \omega t-\theta_{0} \omega N_{r} \sin \omega t+N_{0}
\end{aligned}
$$

Using the same data processing method as for the sway motion, the hydrodynamic coefficients can be calculated as follows:

$$
\left\{\begin{array}{l}
Y_{\dot{r}}=-\frac{F_{\lambda}}{\theta_{0} \omega^{2}}, Y_{r}=-\frac{F_{\mu}}{\theta_{0} \omega} \\
N_{\dot{r}}=-\frac{M_{\lambda}}{\theta_{0} \omega^{2}}, N_{r}=-\frac{M_{\mu}}{\theta_{0} \omega}
\end{array}\right.
$$

Similarly, the corresponding hydrodynamic coefficients can be obtained by substituting the test results into Eq. (28) through linear fitting. The hydrodynamic coefficients obtained by the dimensionless treatment are summarised in Table 10. The test results are shown in Fig. 12.

Tab. 10. Hydrodynamic coefficients of the ship model in yaw test

\begin{tabular}{|c|c|c|c|}
\hline $\begin{array}{c}\text { Hydrodynamic } \\
\text { coefficient }\end{array}$ & Test value $\times \mathbf{1 0}^{-\mathbf{2}}$ & $\begin{array}{c}\text { Value from real } \\
\text { ship } \times \mathbf{1 0}^{-2}[\mathbf{3 0}]\end{array}$ & $\begin{array}{c}\text { Percentage of } \\
\text { difference/\% }\end{array}$ \\
\hline$Y_{r}^{\prime}$ & 1.201 & 1.242 & $3.30 \%$ \\
\hline$N_{r}^{\prime}$ & -0.721 & -0.703 & $2.56 \%$ \\
\hline$Y_{\dot{r}}^{\prime}$ & -0.156 & -0.159 & $1.89 \%$ \\
\hline$N_{\dot{r}}^{\prime}$ & -0.172 & -0.158 & $8.86 \%$ \\
\hline
\end{tabular}

It can be seen from the test results that, compared with the empirical equation and the test results of the circulation tank, the maximum deviation of the test device proposed in this paper is $8.86 \%$. The deviation result is less than $10 \%$, and the deviation range meets the technical requirements of general practical engineering. The results show that the $6 \mathrm{DoF}$ parallel mechanism test platform proposed in this paper can accurately measure 8 typical hydrodynamic coefficients in the horizontal plane of the ship.
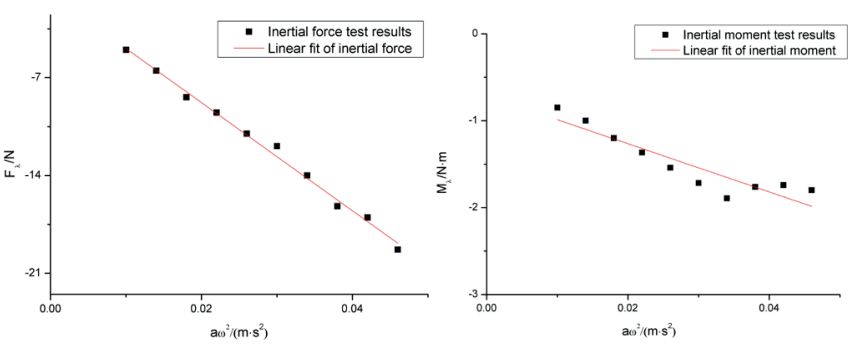

Fig. 11. The test results of sway test
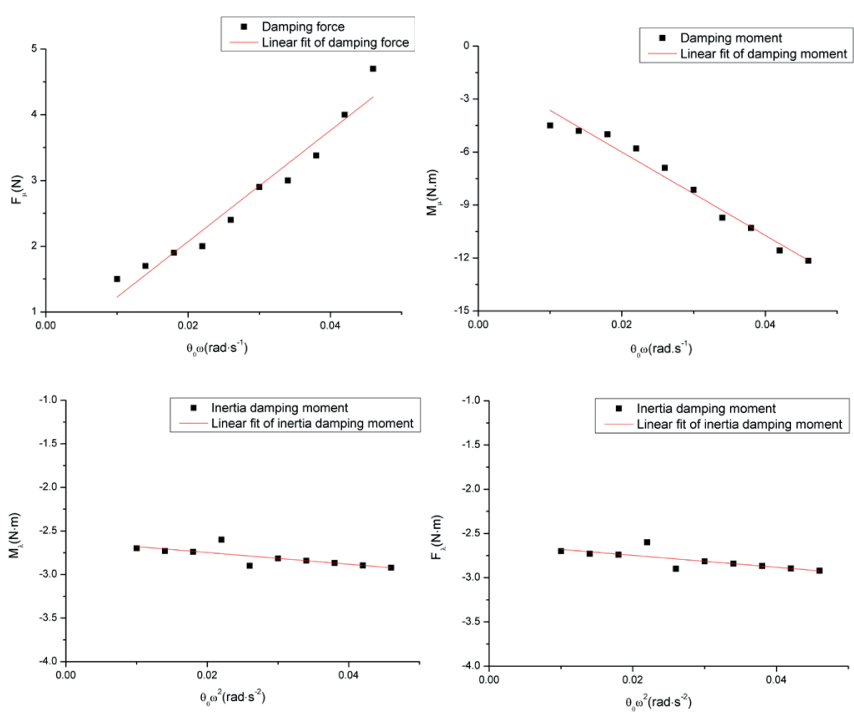

Fig. 12. The test results of yaw test

\section{CONCLUSIONS}

In this paper, a $6 \mathrm{DoF}$ parallel mechanism test platform is presented which can be used in the hydrodynamic testing of ships. With this new device, a ship model can be forced to generate $6 \mathrm{DoF}$ motions independently or harmoniously, which cannot be accomplished by traditional plane motion mechanisms (PMM) or circulating flume tests alone. Firstly, this paper designed the test platform of the $6 \mathrm{DoF}$ parallel mechanism and selected the type of $6 \mathrm{DoF}$ force/torque sensor. Secondly, the principle of measuring the hydrodynamic coefficients by using a mathematical model was described. Finally, eight typical hydrodynamic coefficients of a ship model were measured by means of experimental research. The test results show that the new hydrodynamic coefficient test platform can accurately measure the 10 typical hydrodynamic coefficients of the ship's horizontal motion, and the deviation is less than $10 \%$, which can meet the needs of practical engineering.

The advantage of this model is that only one test platform can drive the ship model to move with six degrees of freedom, and some coupling hydrodynamic coefficients that are difficult to measure can also be measured. In future work, we will use this model to measure the hydrodynamic coefficients of 
coupled hydrodynamic coefficients and the hydrodynamic coefficients of an underwater vehicle model.

\section{ACKNOWLEDGEMENTS}

This project is partially supported by the National Natural Science Foundation of China (No. 51679101, No. 51979117, No. 51405117) and Fundamental Research Funds for the Central Universities (No. 0118140031, No. 0118140032).

\section{REFERENCES}

1. Muscari R., Dubbioso G., Viviani M., Mascio A. D. (2017): Analysis of the asymmetric behavior of propeller-rudder system of twin screw ships by CFD. Ocean Engineering, $143,269-281$.

2. Lidtke A. D., Turnock S. R., Downes J. (2017): Hydrodynamic design of underwater gliders using $k-k L-\omega R A N S$ transition model. IEEE Journal of Oceanic Engineering, 43(2), 356-368.

3. Chen J., Wei J., Yang L. (2018): Hydrodynamic optimization of appendages on ROPAX by using CFD and model tests. Ship Building of China, 59(2), 33-41.

4. Jianglong S., Haiwen T., Yongnian C., De X., Jiajian Z. (2016): A study on trim optimization for a container ship based on effects due to resistance. Journal of Ship Research, 60(1), 30-47.

5. Haiwen T., Yunfei Y. et al. (2018): A modified admiralty coefficient for estimating power curves in EEDI calculations. Ocean Engineering, 150, 309-317.

6. Chuang Z., Steen S. (2013): Speed loss of a vessel sailing in oblique waves. Ocean Engineering, 64, 88-99.

7. Lee P.-M., Jun B.-H., Kim K.-H., Lee J.-H., Aoki T., Hyakudome T. (2007: Simulation of an inertial acoustic navigation system with range aiding for an autonomous underwater vehicle. IEEE Journal of Oceanic Engineering, 32(2), 327-345.

8. Li B., Su T.-C. (2016): Nonlinear heading control of an autonomous underwater vehicle with internal actuators. Ocean Engineering, 125, 103-112.

9. Kim J.-Y., Kim K.-H., Choi H.-S., Seong W.-J., Lee K.-Y. (2002): Estimation of hydrodynamic coefficients for an AUV using nonlinear observers. IEEE Journal of Oceanic Engineering, 27(4), 830-840.

10. Mansoorzadeh S., Javanmard E. (2014): An investigation of free surface effects on drag and lift coefficients of an autonomous underwater vehicle (AUV) using computational and experimental fluid dynamics methods. Journal of Fluids \& Structures, 51(1), 161-171.

11. Gala F. L., Dubbioso G., Ortolani F., et al. (2012): Preliminary evaluation of control and manoeuvring qualities for the AUTODROP-UUV vehicle. IFAC Proceedings Volumes, 45(27), 132-137.

12. Li G. (2011): Numerical and experimental research on hydrodynamic characters of shuttle submersible. Harbin Engineering University, Harbin, 2011.

13. Avila J. P. J., Adamowski J. C. (2011): Experimental evaluation of the hydrodynamic coefficients of a ROV through Morison's equation. Ocean Engineering, 38(17), 2162-2170.

14. Xu F., Zou Z. J., Yin J. C., et al. (2013): Identification modeling of underwater vehicles' nonlinear dynamics based on support vector machines. Ocean Engineering, 67, 68-76.

15. Zhao J.-X. (2011): The hydrodynamic performance calculation and motion simulation of an AUV with appendages. Harbin Engineering University, Harbin.

16. Pang Y.-J., Wang Q.-Y., Li W.-P. (2017): Model test study of influence of propeller and its rotation on hydrodynamics of submarine maneuverability. Journal of Harbin Engineering University, 38(1), 109-114.

17. Kijima K., Nakiri Y. (1990): On a numerical simulation for predicting of ship manoeuvring performance. 19th International Towing Tank Conference, Madrid, Spain, Vol. 2, 559-568.

18. Maekawa K., Shuto C., Karasuno K., Nonaka K. (1999): Estimation of added mass coefficients $m x^{\prime}, m y$ ' by using CFD through oblique towing test with constant acceleration. Journal of Kansai Society of Naval Architects Japan, 232, $55-61$.

19. Kijima K., Nakari Y., Furukawa Y. (2000): On a prediction method for ship manouevrability. International Workshop on Ship Manoeuvrability at the Hamburg Ship Model Basin, Hamburg, Germany, pp. 536-543.

20. Petersen, J. B., Lauridsen, B. (2000): Prediction of hydrodynamic forces from a database of manoeuvring derivatives. MARSIM 2000, Orlando, FL, USA, pp. 401-420.

21. Yang C.-F., Wu B.-S., Shen H.-C. (2006): Analysis of experiment validation for full- ship maneuverability hydrodynamic forces prediction. Journal of Ship Mechanics, 10(4), 559-568.

22. Gao T., Wang Y.-X., Pang Y.-J., Chen Q.-L., Tang Y.-G. (2018): A time-efficient CFD approach for hydrodynamic 
coefficient determination and model simplification of submarine. Ocean Engineering, 154, 16-26.

23. Stewart D. (1966): A platform with six degrees of freedom. Aircraft Engineering and Aerospace Technology, 38(4), 30-35.

24. Yurt S. N., Ozkol I., Hajiyev C. (2004): Error analysis and motion determination of a flight simulator. Aircraft Engineering and Aerospace Technology, 76(2), 185-192.

25. Landry S. J., Jacko J. (2012): Pilot Procedure-Following Behavior during Closely Spaced Parallel Approaches. International Journal of Human-Computer Interaction, 28(2), 131-139.

26. Phoemsapthawee S., Le Boulluec M. (2013): A potential flow based flight simulator for an underwater glider. Journal of Marine Science and Application, 12(1), 112-121.

27. Kim G. S. (2007): Design of a six-axis wrist force/moment sensor using FEM and its fabrication for an intelligent robot. Sensors and Actuators A Physical, 133(1), 27-34.

28. Nekrasov V: (2019): Mean-Square Non-Local Stability of Ship in Storm Conditions of Operation. Polish Maritime Research, 26(4), 6-15.

29. Kun D., Yunbo L. (2019): Manoeuvring Prediction of KVLCC2 with Hydrodynamic Derivatives Generated by a Virtual Captive Model Test. Polish Maritime Research, 26(4), 16-26.

30. CSSRC (2018): Ship test report for KELC tank ship. Report (Wuhan China), pp. 17-24.

\section{CONTACT WITH THE AUTHORS}

Yun Lu

e-mail:hustluyun@hust.edu.cn

Jinbo Wu

e-mail:2373751844@qq.com

\section{Weijia Li}

e-mail:61525963@qq.com

Yaozhong Wu

e-mail:no2kenk98@sina.comm

School of Naval Architecture and Ocean Engineering Huazhong University of Science and Technology

Luoyu Road 1037\#

Hongshan Distinct

430074 Wuhan

CHINA 